\title{
MiR-323 Inhibits Prostate Cancer Vascularization Through Adiponectin Receptor
}

\author{
Qiruo Gao Xudong Yao Junhua Zheng \\ Department of Urology, Shanghai 10th People's Hospital, Tongji University School of Medicine, \\ Shanghai, China
}

\section{Key Words}

Prostate cancer $(\mathrm{PC}) \cdot$ Adiponectin $\cdot$ Adiponectin receptor 1 (AdipoR1) • miR-323 • Vascular endothelial growth factor A (VEGF-A)

\begin{abstract}
Background/Aims: The current treatments fail to provide satisfactory cure for aggressive prostate cancers (PCs). Hence, further comprehension of PC metastasis is highly appreciated for improving the levels of therapy. We have previously shown that Adiponectin reduces the levels of vascular endothelial growth factor A (VEGF-A) in PCs to suppress tumor-associated neovascularization, possibly through AMPK/mTor signaling. Here, we studied the regulation of Adiponectin signaling in PCs. Methods: We analyzed the levels and correlation of Adiponectin receptor 1 (AdipoR1) and microRNA-323 (miR-323) in the PC specimen, compared to the paired normal prostate tissue. We analyzed the binding of miR-323 to the 3'UTR of AdipoR1 mRNA and its effects on AdipoR1 translation by bioinformatics analysis and by luciferasereporter assay, respectively. We modified miR-323 levels in PC cells, and examined the effects on the expression of AdipoR1 and VEGF-A, as well as on vessel formation in a human umbilical vein endothelial cells (HUVECs) transwell collagen gel assay. Results: We detected significantly lower levels of AdipoR1 and significantly higher levels of miR-323 in PC specimen. Moreover, the levels of AdipoR1 and miR-323 are inversely correlated. Moreover, miR-323 was found to bind to the $3^{\prime} U T R$ of AdipoR1 mRNA to inhibit its translation. Overexpression of miR-323 in PC cells decreased AdipoR1 protein levels, whereas inhibition of miR-323 increased AdipoR1 protein levels, without affecting AdipoR1 transcripts. Moreover, overexpression of miR-323 increased the levels of VEGF-A and the vessel formation by HUVECs, while inhibition of miR323 decreased the levels of VEGF-A and the vessel formation by HUVECs. Conclusion: Our data demonstrate that miR-323 may increase VEGF-A-mediated cancer vascularization in PC cells through AdipoR1 suppression.
\end{abstract}




\section{Cellular Physiology Cell Physiol Biochem 2015;36:1491-1498 \begin{tabular}{l|l|l} 
DOI: 10.1159/000430313 & C 2015 S. Karger AG, Basel
\end{tabular} \begin{tabular}{l|l} 
and Biochemistry Published online: July 06, 2015 & www.karger.com/cpb
\end{tabular} \\ Gao/Yao/Zheng: MiR-323 Inhibits AdipoR1 in PC}

\section{Introduction}

In the population of aged men, prostate cancer (PC) is a common malignant tumor, and mostly grow slowly. However, aggressive PCs capable of invasion do occur, in which the primary cancer cells migrate and seed to distal organs and lymph nodes [1-4]. Since the current treatments upon PCs are not sufficient to provide satisfactory therapy for aggressive PCs, further comprehension of PC metastasis is highly appreciated [1-7].

Cancer metastasis greatly associates with cancer-related vascularization [8-12]. Tumor cells release soluble angiogenic factors to enhance new vessel formation and restructure of existing vessels to allow tumors to outgrow and migrate [8-12]. The angiogenic events are coordinately regulated by pro-angiogenic and anti-angiogenic factors [8-12]. The most potent pro-angiogenic factor is vascular endothelial growth factor A (VEGF-A) [13-17], which has also been shown to play a critical role in both embryonic and adult vascularization. Of note, a pivotal role of VEGF-A in the cancer-associated vascularization in PC has been acknowledged and used as a therapeutic target [8-12]. Adiponectin is a hormone derived from adipose tissue. Adiponectin has a well-established function in prohibiting development of diabetes, atherosclerosis, inflammation and cell replication. Adiponectin has two receptors, Adiponectin receptor I (AdipoR1) and Adiponectin receptor II (AdipoR2) [18-20], which distribute widely among different organs. Recently, the anticarcinogenic effects of adiponectin have been appreciated [21-23]. However, the underlying mechanisms are not clear. We have shown that Adiponectin may inhibit VEGF-A-mediated cancer neovascularization in PC cells through activation of AMPK/TSC2 and suppression of mTor [24]. Nevertheless, the regulation of Adiponectin receptor signaling in PC cells are still unknown.

MicroRNAs (miRNAs) are a class of small, non-coding RNAs that regulate various biological processes [25-27]. Interestingly, bioinformatics approaches have predicted onethird of all mammalian genes to be targeted and regulated by miRNAs [25-27]. Previous studies have shown that aberrant miRNA expression could impact normal biological processes, resulting in PC initiation and progression [28-30]. However, a role of miR-323 in the carcinogenesis of PC has not been studied.

In the current study, we examined the regulation of AdipoR1 signaling in PC cells. We detected significantly lower levels of AdipoR1 and significantly higher levels of miR-323 in PC specimen. Moreover, the levels of AdipoR1 and miR-323 are inversely correlated. Moreover, miR-323 was found to bind to the 3'UTR of AdipoR1 mRNA to inhibit its translation. Overexpression of miR-323 in PC cells decreased AdipoR1 protein levels, whereas inhibition of miR-323 increased AdipoR1 protein levels, without affecting AdipoR1 transcripts. Moreover, overexpression of miR-323 increased the levels of VEGF-A and the vessel formation by HUVECs, while inhibition of miR-323 decreased the levels of VEGF-A and the vessel formation by HUVECs.

\section{Materials and Methods}

\section{Patient tissue specimens}

A total of 24 resected specimens from PC patients were collected for this study. OS specimen was compared with the paired normal prostate tissue (NPT) from the same patient. All specimens had been histologically and clinically diagnosed at the Tongji University School of Medicine from 2009 to 2014. For the use of these clinical materials for research purposes, prior patient's consents and approval from the Institutional Research Ethics Committee were obtained.

Human PC cell lines

PC3, DU-145, CA-HPV-10 and MDA-PCa-2b are 4 commonly used human prostate cancer line, and were all purchased from ATCC (American Type Culture Collection, Manassas, VA, USA). These cell lines were cultured in Dulbecco's Modified Eagle's Medium (DMEM, Invitrogen, Carlsbad, CA, USA) supplemented with 20\% 


\section{Cellular Physiology Cell Physiol Biochem 2015;36:1491-1498 \begin{tabular}{ll|l} 
DOI: 10.1159/000430313 & $\begin{array}{l}\text { O 2015 S. Karger AG, Basel } \\
\text { www.karger.com/cpb }\end{array}$ \\
\hline
\end{tabular} \\ Gao/Yao/Zheng: MiR-323 Inhibits AdipoR1 in PC}

fetal bovine serum (Invitrogen). PC3 cells were transfected with a miR-323 construct, or an antisense (as) of miR-323, or a scramble sequence (scr) as a control, and after which the transfected cells were purified based on green fluorescence protein (GFP) to generate PC3-miR-323, or PC3-as-miR-323 cells or PC3-scr, respectively. MiR-323 sequence: 5'-AUGUAAUGAGACGGUUCUUUUUU-3', miR-323 antisense sequence: 5'-AAAAAAGAACCGUCUCAUUACAU-3', control sequence: 5'-AAUAGCAUCGAAAAGUCCGG-3'. Transfection was performed with Lipofectamine 2000 reagent (Invitrogen), according to the manufacturer's instructions.

\section{HUVEC transwell collagen gel assay}

Human umbilical vein endothelial cells (HUVECs) were grown in M-200 supplemented medium with low serum growth supplement (Invitrogen). HUVEC endothelial cells were embedded in a collagen gel, and plated on a 24-well culture plate. The plate was kept at $37^{\circ} \mathrm{C}$ in a $\mathrm{CO}_{2}$ incubator for $10-15$ minutes to make the collagen polymerize, and then the same number of prostate cancer cells (with miR-323 modification) was added to the transwell and put onto the plate. The plate with transwell was returned to the $\mathrm{CO}_{2}$ incubator. Media was replenished every day, and culture images were taken after 3 days.

\section{Luciferase-reporter activity assay}

Luciferase-reporters were successfully constructed using molecular cloning technology. Target sequence was inserted into pGL3-Basic vector (Promega, Madison, WI, USA) to obtain pGL3-AdipoR13'UTR, which contains the miR-323 binding sequence (AdipoR1-3'UTR sequence). PC3-miR-323, or PC3scr, or PC3-antisense (as)-miR-323 cells were seeded in 24-well plates for 24 hours, after which they were transfected with $1 \mu \mathrm{g}$ of Luciferase-reporter plasmid per well using PEI Transfection Reagent. Then luciferase activities were measured using the dual-luciferase reporter gene assay kit (Promega), according to the manufacturer's instructions.

\section{Quantitative PCR (RT-qPCR)}

MiRNA and total RNA were extracted from cultured cells with miRNeasy mini kit or RNeasy kit (Qiagen, Hilden, Germany), respectively, and used for cDNA synthesis. Quantitative PCR was performed in duplicates with QuantiTect SYBR Green PCR Kit (Qiagen). All primers were purchased from Qiagen. Data were analyzed using $2-\Delta \Delta \mathrm{Ct}$ method for quantification of the relative mRNA expression levels. Values of genes were normalized against $\alpha$-tubulin, and then compared to controls.

\section{Western blot}

The protein was extracted from the resected PC specimen or adjacent normal prostate tissue (NPT), or cultured cells. Primary antibodies were anti-AdipoR1 and anti- $\alpha$-tubulin (Cell Signaling, San Jose, CA, USA). $\alpha$-tubulin was used as a protein loading control. Secondary antibodies were HRP-conjugated anti-rabbit, and were all purchased from Jackson ImmunoResearch Labs (West Grove, PA, USA). The protein levels were first normalized to $\alpha$-tubulin, and then normalized to control, quantified by NIH ImageJ software (Bethesda, MA, USA).

\section{ELISA assay}

The concentration of VEGF-A in the conditioned media from cultured cells was determined by a human VEGF-A ELISA kit (Raybio, Norcross, GA, USA). ELISAs were performed according to the instructions of the manufacturer. Briefly, the collected condition medium was added to a well coated with VEGF-A polyclonal antibody, and then immunosorbented by biotinylated monoclonal anti-human VEGF-A antibody at room temperature for 2 hours. The color development catalyzed by horseradish peroxidase was terminated with $2.5 \mathrm{~mol} / 1$ sulfuric acid and the absorption was measured at $450 \mathrm{~nm}$. The protein concentration was determined by comparing the relative absorbance of the samples with the standards.

\section{Statistical analysis}

All statistical analyses were carried out using the SPSS 18.0 statistical software package. All values are depicted as mean \pm standard deviation and are considered significant if $\mathrm{p}<0.05$. All data were statistically analyzed using one-way ANOVA with a Bonferoni correction, followed by Fisher's Exact Test to compare two groups. Bivariate correlations were calculated by Spearman's rank correlation coefficients.

\section{KARGER}




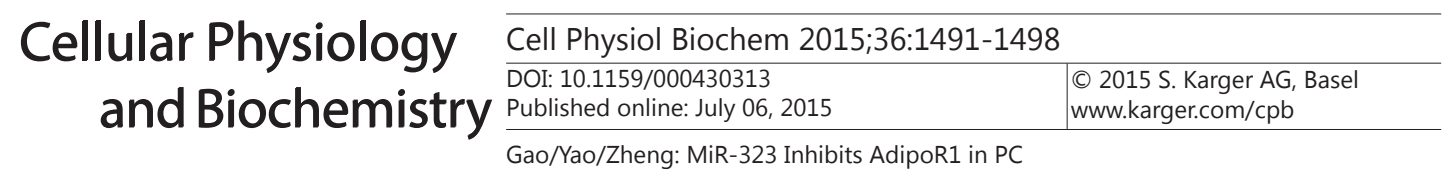

\section{Results}

Low levels of AdipoR1 and high levels of miR-323 are inversely correlated in PC

We examined the AdipoR1 levels and the miR-323 levels in the resected PC specimens from 24 patients, and compared to the paired normal prostate tissue (NPT). We detected significantly lower levels of AdipoR1 by Western blot (Fig. 1A), and significantly higher levels of miR-323 by RT-qPCR (Fig. 1B), respectively, in the PC specimen compared to control NPT. Moreover, a strong inverse correlation was detected between AdipoR1 and miR-323 (Fig. 1C, $\mathrm{R}=-0.85 ; \mathrm{p}<0.0001$ ), suggesting a causal relationship.

\section{MiR-323 targets 3'UTR of AdipoR1 to inhibit its expression}

Based on our findings in the patients' samples, we performed bioinformatics analysis of AdipoR1 target sequence, which show that miR-323 binds to 3'UTR of AdipoR1 mRNA at $601^{\text {th }}-608^{\text {th }}$ base site (Fig. 2A). In order to examine whether its binding may be functional, we used a human PC cell line, PC3, in our study. We transfected PC3 cells with either miR323 or antisense for miR-323 (as-miR-323). PC3 cells were also transfected with a scramble sequence as a control (scr). Modulation of miR-323 levels in PC3 cells was confirmed by RTqPCR (Fig. 2B). Then, PC3-miR-323, PC3-scr and PC3-as-miR-323 cells were transfected with $1 \mu \mathrm{g}$ of AdipoR1-3'UTR Luciferase-reporter plasmid. We found that the luciferase activities in PC3-as-miR-323 cells were significantly higher than the control scr, while the luciferase activities in PC3-miR-323 cells were significantly lower than the control scr (Fig. 2C). These data suggest that miR-323 targets 3'UTR of AdipoR1 to inhibit its translation.

MiR-323 decreases AdipoR1 protein but does not affect its transcription

Since AdipoR1 is necessary for the inhibitory effects on VEGF-A by Adiponectin in PC cells, we were thus prompted to evaluate whether AdipoR1 may be regulated by miR323 in PC cells. We found that although the AdipoR1 transcripts did not change by miR323 (Fig. 3A), the protein levels of AdipoR1 in miR-323-overexpressing PC3 cells was significantly decreased, while the protein levels of AdipoR1 in miR-323-depleted PC3 cells was significantly increased (Fig. 3B-C). These data suggest that the translation of AdipoR1 in PC cells may be regulated by miR-323.

MiR-323 inhibits VEGF-A production in PC cells

Then we examined whether the effects of miR-323 on AdipoR1 may result in changes in regulation of VEGF-A production in PC cells. We thus analyzed VEGF-A levels in miR-

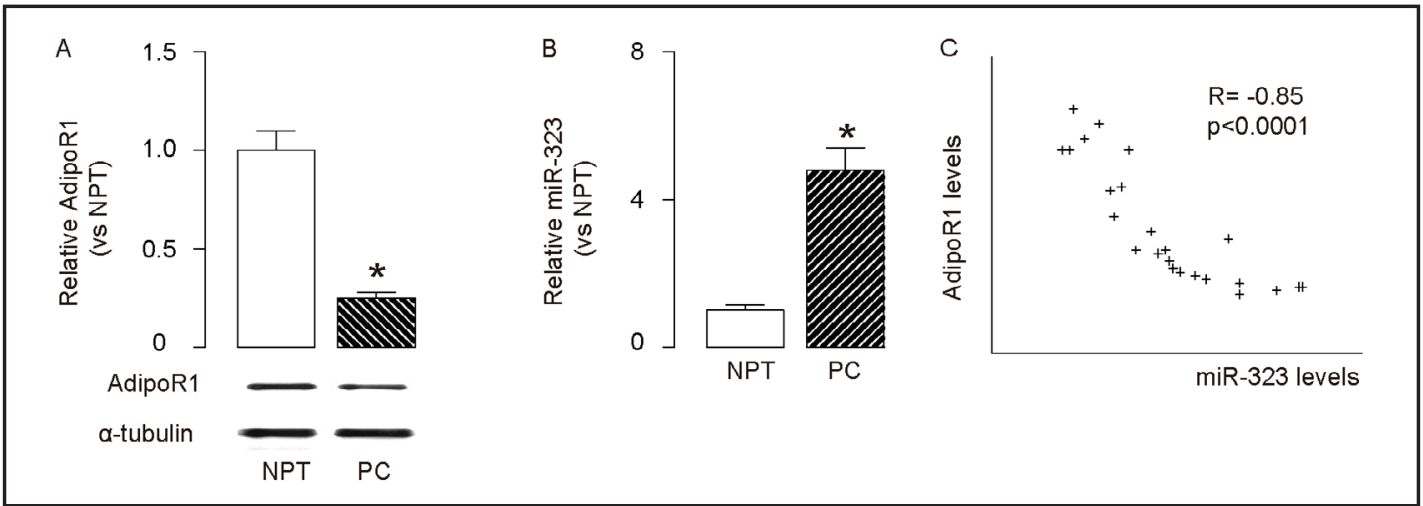

Fig. 1. Low AdipoR1 and high miR-323 are inversely correlated in PC. We examined the AdipoR1 levels and miR-323 levels in the resected PC specimens from 24 patients, and compared to the paired normal prostate tissue (NPT). (A-B) Examination of AdipoR1 levels by Western blot (A), and miR-323 levels by RT-qPCR (B). (C) A strong inverse correlation was detected between AdipoR1 and miR-323 in PC specimen (R= -0.85 ; $p<$ $0.0001){ }^{*}: \mathrm{p}<0.05$. 
A predicted binding of miR-323 to AdipoR1 3'-UTR

5' UUUUUucuUggagaguaAugua 3 ' AdipoR1 3'-UTR (601-608)

3' UCUCCAGCUGGCACAUUACAC 5 , miR-323
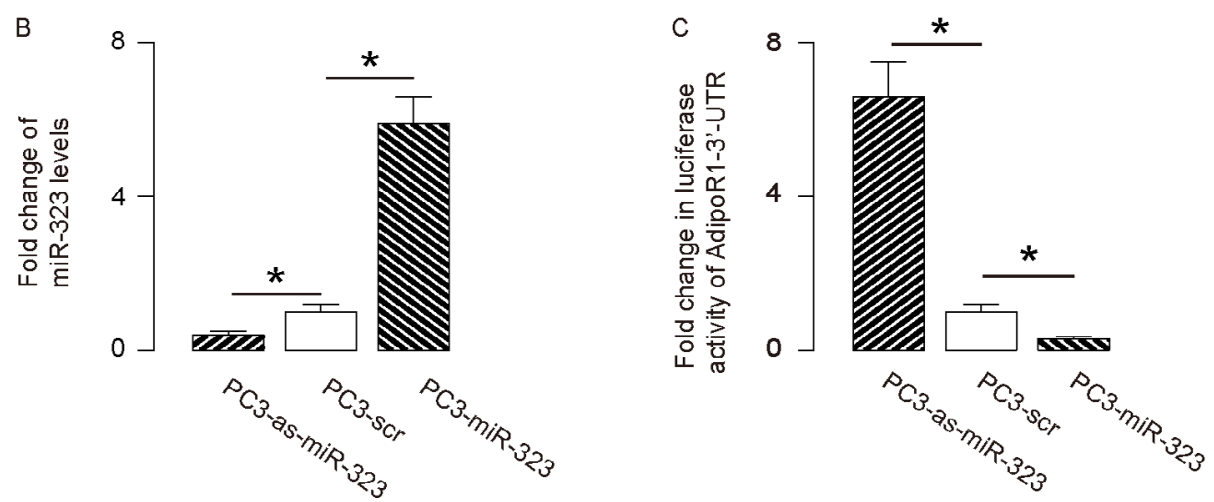

Fig. 2. MiR-323 targets 3'UTR of AdipoR1 to inhibit its expression. (A) Bioinformatics analyses of AdipoR1 target sequence show that the miR-323 binds to the 3'UTR of AdipoR1 mRNA. (B-C) We overexpressed or inhibited miR-323 expression in a human PC cell line, PC3, to obtain PC3-miR-323 and PC3-as-miR-323 cells, respectively. The cells transduced with a control plasmid carrying a scramble sequence (PC3-scr). (B) RT-qPCR on miR-323. (C) PC3-miR-323, PC3-scr and PC3-as-miR-323 cells were transfected with $1 \mu \mathrm{g}$ of AdipoR1-3'UTR luciferase-reporter plasmid. The luciferase activities in these cells were then evaluated. *: $\mathrm{p}<0.05$.

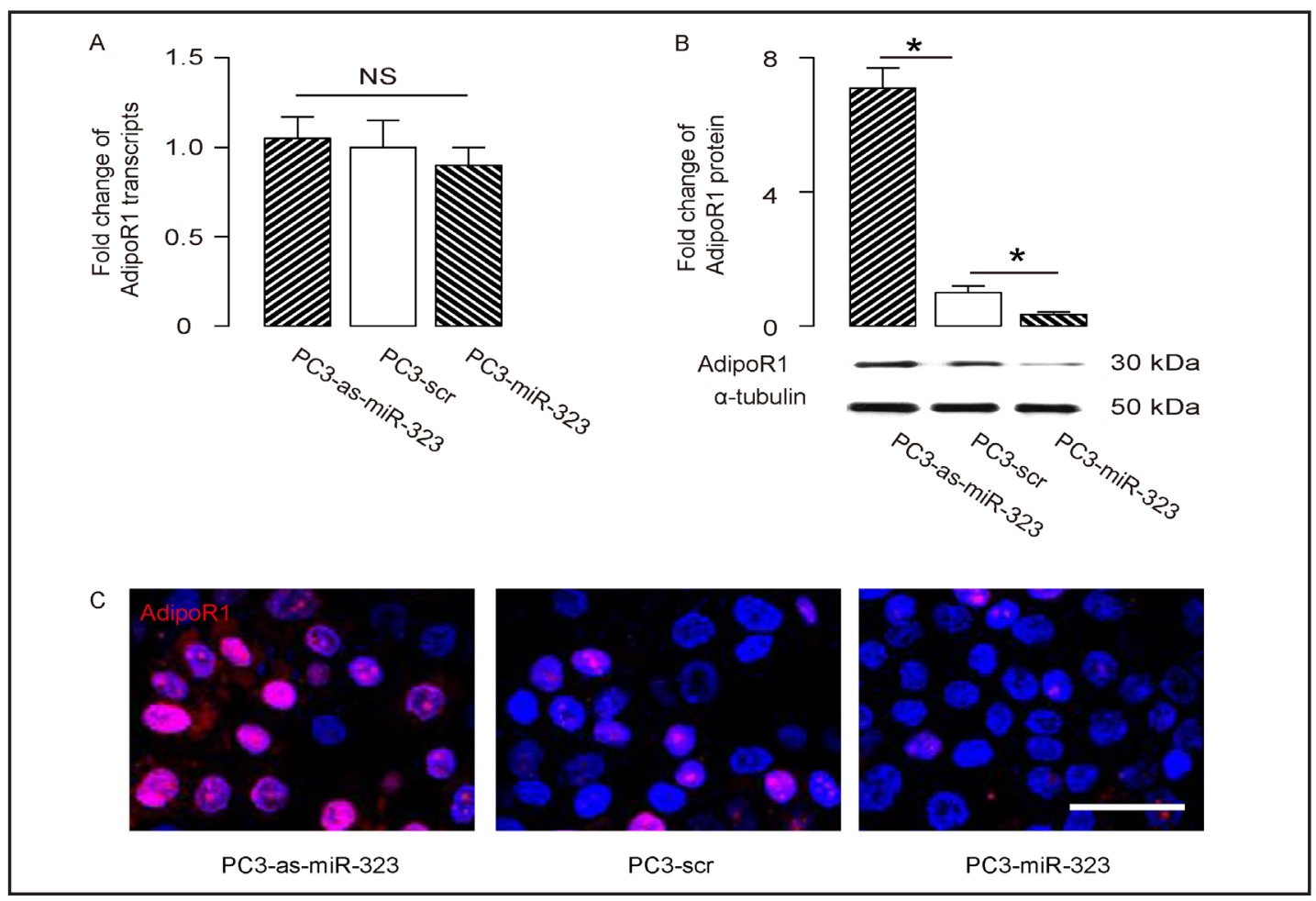

Fig. 3. MiR-323 decreases AdipoR1 levels in PC cells. (A) RT-qPCR on AdipoR1. (B) Western blot images and quantification for AdipoR1. (C) Immunocytochemistry for AdipoR1. *: p<0.05. NS: non-significant. Scale bar is $20 \mu \mathrm{m}$. 
Fig. 4. MiR-323 inhibits VEGF-A production in PC cells. (A-B) VEGF-A levels in miR-323-modified PC3 cells were examined by RT-qPCR (A), and by ELISA on the secreted protein in the conditioned media (B). (C-D) In a HUVEC tube formation assay, the tube formation by HUVECs co-cultured with miR-323-modified PC3 cells was shown by quantification (C), and by representative images (D). *: p<0.05. Scale bar is $30 \mu \mathrm{m}$.
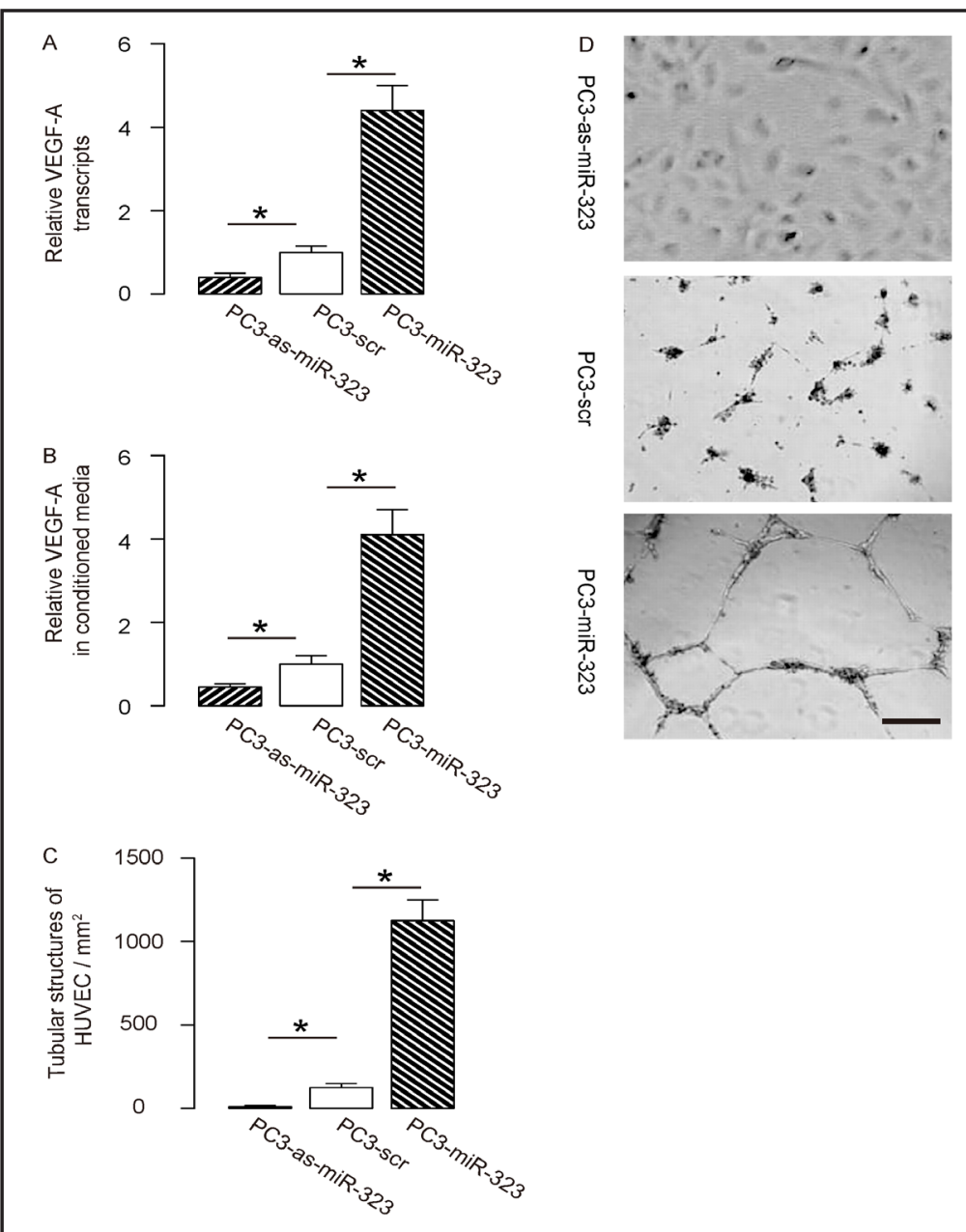

Fig. 5. Schematic of the model. MiR-323 may increase VEGF-A-mediated cancer vascularization in PC cells through AdipoR1 suppression.

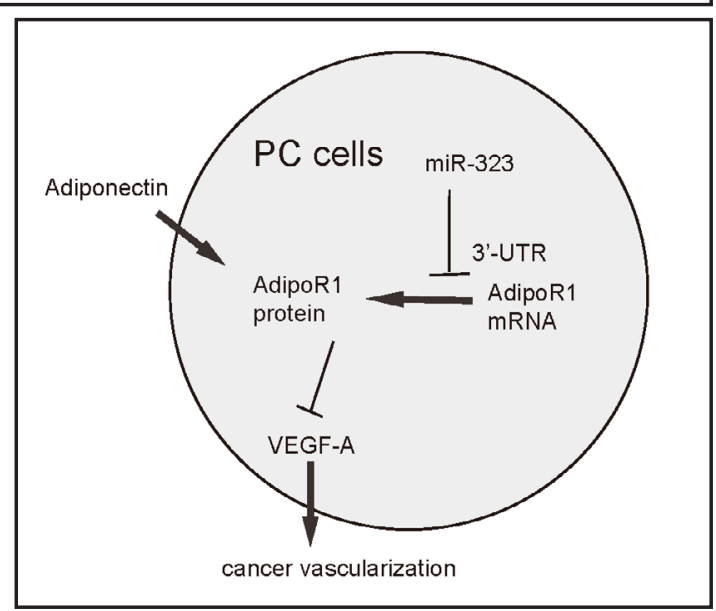

323-modified PC3 cells. We found that overexpression of miR-323 in PC3 cells significantly increased production of VEGF-A, while miR-323 depletion significantly decreased VEGF-A, by RT-qPCR (Fig. 4A), and by ELISA on the secreted protein in the conditioned media (Fig. 4B). In a HUVEC tube formation assay, we found that HUVECs substantially increased their tube formation when co-cultured with miR-323-overexpressing PC3 cells, and substantially decreased their tube formation when co-cultured with miR-323-depleted PC3 cells (Fig. 4C-D). Our study thus demonstrate that miR-323 may increase VEGF-A-mediated cancer vascularization in PC cells through AdipoR1 suppression (Fig. 5). 


\section{Cellular Physiology Cell Physiol Biochem 2015;36:1491-1498 \begin{tabular}{l|l|l} 
DOI: 10.1159/000430313 & $\begin{array}{l}\text { O 2015 S. Karger AG, Basel } \\
\text { www.karger.com/cpb }\end{array}$ \\
\hline
\end{tabular} \\ Gao/Yao/Zheng: MiR-323 Inhibits AdipoR1 in PC}

\section{Discussion}

Essential roles of miRNAs and Adiponectin have been well-established in the neovascularization-associated carcinogenesis, whereas the relationship between miR-323 and Adiponectin receptor signaling in PC cells has not been reported. In our recent report, we found that overexpression of adiponectin in prostate cancer cells decreased production of VEGF-A, while adiponectin depletion increased VEGF-A. Furthermore, adiponectin seemed to activate AMPK/TSC2 to inhibit mTor-mediated activation of VEGF-A in PC3 cells [24]. However, the regulation of Adiponectin receptor signaling in PC cells are not understood.

In the current study, we aimed to answer this question. Then we used 4 human PC cell lines to study the mechanism. Since we obtained similar results from these 4 PC lines, we only showed our data using PC3 cells. In a previous bioinformatics screening, we identified several candidate miRNAs that may target 3'UTR on AdipoR1 mRNA. From these candidates, we found that miR-323 specifically increased in PC, compared to control tissue. Moreover, we detected strong inverse correlation between AdipoR1 and miR-323 in PC specimen, suggesting presence of a regulatory relationship.

We thus examined whether miR-323 may inhibit the expression of AdipoR1. We either overexpressed or inhibited miR-323 expression in PC cells, which decreased or increased the protein levels of AdipoR1, respectively, without affecting AdipoR1 transcripts. These data suggest that AdipoR1 translational controls in PC cells may be regulated by miR-323, which is consistent with the function of miRNAs. Moreover, using luciferase reporter assay, we found that the binding of miR-323 to 3'UTR on AdipoR1 mRNA resulted in translation inhibition. Taken together, these findings strongly demonstrate a critical role of miR-323 in regulating AdipoR1 translation, rather than in regulating the degradation of AdipoR1 mRNA.

In addition, we found that the modification of miR-323 levels in PC cells significantly altered VEGF-A levels, as a consequence of adaptation of Adiponectin-stimulated signal transduction. Our findings thus highlight miR-323/AdipoR1 axis as a novel target for regulating the cancer-associated neovascularization. These data suggest that modulation of miRNAs in PC cells may indirectly regulate VEGF-A-mediated cancer vessel formation, which could potentially reduce the adverse effects of VEGF-A antagonists in patients.

\section{Disclosure Statement}

The authors have declared that no competing interests exist.

\section{Reference}

1 Saylor PJ: Prostate cancer: The androgen receptor remains front and centre. Nat Rev Clin Oncol 2013;10:126-128.

2 Alva A, Hussain M: The changing natural history of metastatic prostate cancer. Cancer J 2013;19:19-24.

3 Beltran H, Rubin MA: New strategies in prostate cancer: Translating genomics into the clinic. Clin Cancer Res 2013;19:517-523.

$4 \quad$ Xin L: Cells of origin for cancer: An updated view from prostate cancer. Oncogene 2013;32:3655-3663.

5 Lin R, Feng J, Dong S, Pan R, Zhuang H, Ding Z: Regulation of autophagy of prostate cancer cells by betacatenin signaling. Cell Physiol Biochem 2015;35:926-932.

6 Li T, Zhao X, Mo Z, Huang W, Yan H, Ling Z, Ye Y: Formononetin promotes cell cycle arrest via downregulation of akt/cyclin d1/cdk4 in human prostate cancer cells. Cell Physiol Biochem 2014;34:13511358.

7 Gillies K, Wertman J, Charette N, Dupre DJ: Anterograde trafficking of cxcr4 and ccr2 receptors in a prostate cancer cell line. Cell Physiol Biochem 2013;32:74-85.

8 Antonarakis ES, Carducci MA: Targeting angiogenesis for the treatment of prostate cancer. Expert Opin Ther Targets 2012;16:365-376.

9 Kluetz PG, Figg WD, Dahut WL: Angiogenesis inhibitors in the treatment of prostate cancer. Expert Opin Pharmacother 2010;11:233-247. 


\section{Cellular Physiology Cell Physiol Biochem 2015;36:1491-1498 \begin{tabular}{l|l|l|}
\hline DOI: 10.1159/000430313 & O 2015 S. Karger AG, Basel \\
and Biochemistry & Published onlin: July 06, 2015 &
\end{tabular} \\ Gao/Yao/Zheng: MiR-323 Inhibits AdipoR1 in PC}

10 Aragon-Ching JB, Dahut WL: Vegf inhibitors and prostate cancer therapy. Curr Mol Pharmacol 2009;2:161168.

11 Delongchamps NB, Peyromaure M: The role of vascular endothelial growth factor in kidney and prostate cancer. Can J Urol 2007;14:3669-3677.

12 Delongchamps NB, Peyromaure M, Dinh-Xuan AT: Role of vascular endothelial growth factor in prostate cancer. Urology 2006;68:244-248.

13 Ferrara N: Vascular endothelial growth factor. Arterioscler Thromb Vasc Biol 2009;29:789-791.

14 Otrock ZK, Makarem JA, Shamseddine AI: Vascular endothelial growth factor family of ligands and receptors: Review. Blood Cells Mol Dis 2007;38:258-268.

15 Nieves BJ, D'Amore PA, Bryan BA: The function of vascular endothelial growth factor. Biofactors 2009;35:332-337.

16 Xiao X, Prasadan K, Guo P, El-Gohary Y, Fischbach S, Wiersch J, Gaffar I, Shiota C, Gittes GK: Pancreatic duct cells as a source of vegf in mice. Diabetologia 2014;57:991-1000.

17 Xiao X, Guo P, Chen Z, El-Gohary Y, Wiersch J, Gaffar I, Prasadan K, Shiota C, Gittes GK: Hypoglycemia reduces vascular endothelial growth factor a production by pancreatic beta cells as a regulator of beta cell mass. J Biol Chem 2013;288:8636-8646.

18 Yamauchi T, Kamon J, Ito Y, Tsuchida A, Yokomizo T, Kita S, Sugiyama T, Miyagishi M, Hara K, Tsunoda M, Murakami K, Ohteki T, Uchida S, Takekawa S, Waki H, Tsuno NH, Shibata Y, Terauchi Y, Froguel P, Tobe K, Koyasu S, Taira K, Kitamura T, Shimizu T, Nagai R, Kadowaki T: Cloning of adiponectin receptors that mediate antidiabetic metabolic effects. Nature 2003;423:762-769.

19 Burgess AP, Vanella L, Bellner L, Gotlinger K, Falck JR, Abraham NG, Schwartzman ML, Kappas A: Heme oxygenase (ho-1) rescue of adipocyte dysfunction in ho-2 deficient mice via recruitment of epoxyeicosatrienoic acids (eets) and adiponectin. Cell Physiol Biochem 2012;29:99-110.

20 Goldstein BJ, Scalia R: Adiponectin: A novel adipokine linking adipocytes and vascular function. J Clin Endocrinol Metab 2004;89:2563-2568.

21 Ren T, Qing Y, Dai N, Li M, Qian C, Yang Y, Cheng Y, Li Z, Zhang S, Zhong Z, Wang D: Apurinic/apyrimidinic endonuclease 1 induced upregulation of fibroblast growth factor 2 and its receptor 3 induces angiogenesis in human osteosarcoma cells. Cancer Sci 2014;105:186-194.

22 Mirabello L, Yu K, Berndt SI, Burdett L, Wang Z, Chowdhury S, Teshome K, Uzoka A, Hutchinson A, Grotmol T, Douglass C, Hayes RB, Hoover RN, Savage SA, National Osteosarcoma Etiology Study G: A comprehensive candidate gene approach identifies genetic variation associated with osteosarcoma. BMC Cancer 2011;11:209.

23 Jang JH: Identification and characterization of soluble isoform of fibroblast growth factor receptor 3 in human saos-2 osteosarcoma cells. Biochem Biophys Res Commun 2002;292:378-382.

24 Gao Q Zheng J, Yao X, Peng B: Adiponectin inhibits vegf-a in prostate cancer cells. Tumour Biol 2015 in press.

25 Sicard F, Gayral M, Lulka H, Buscail L, Cordelier P: Targeting mir-21 for the therapy of pancreatic cancer. Mol Ther 2013;21:986-994.

26 Tavano F, di Mola FF, Piepoli A, Panza A, Copetti M, Burbaci FP, Latiano T, Pellegrini F, Maiello E, Andriulli A, di Sebastiano P: Changes in mir-143 and mir-21 expression and clinicopathological correlations in pancreatic cancers. Pancreas 2012;41:1280-1284.

27 Ali S, Ahmad A, Banerjee S, Padhye S, Dominiak K, Schaffert JM, Wang Z, Philip PA, Sarkar FH: Gemcitabine sensitivity can be induced in pancreatic cancer cells through modulation of mir-200 and mir-21 expression by curcumin or its analogue cdf. Cancer Res 2010;70:3606-3617.

28 Saini S, Majid S, Shahryari V, Arora S, Yamamura S, Chang I, Zaman MS, Deng G, Tanaka Y, Dahiya R: Mirna-708 control of cd44(+) prostate cancer-initiating cells. Cancer Res 2012;72:3618-3630.

29 Jin M, Zhang T, Liu C, Badeaux MA, Liu B, Liu R, Jeter C, Chen X, Vlassov AV, Tang DG: Mirna-128 suppresses prostate cancer by inhibiting bmi-1 to inhibit tumor-initiating cells. Cancer Res 2014;74:4183-4195.

30 Wang LN, Chen WW, Zhang J, Li CY, Liu CY, Xue J, Zhang PJ, Jiang AL: The mirna let-7a1 inhibits the expression of insulin-like growth factor 1 receptor (igf1r) in prostate cancer pc-3 cells. Asian J Androl 2013;15:753-758. 